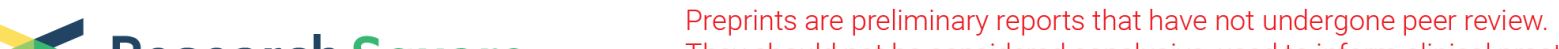 $\begin{array}{ll}\text { Research Square } & \text { They should not be considered conclusive, used to inform clinical practice, } \\ \text { or referenced by the media as validated information. }\end{array}$
}

\section{Ultra-fast Tunable Optoelectronic Half Adder/subtractor based on Photonic Crystal Ring Resonators Covered by Graphene Nanoshells}

\section{Saleh Naghizade}

Islamic Azad University Tabriz Branch

Hamed Saghaei ( $\nabla$ h.saghaei@iaushk.ac.ir)

Islamic Azad University Shahrekord Branch https://orcid.org/0000-0001-5588-3726

\section{Research Article}

Keywords: Photonic crystal microstructure, nonlinear material, optoelectronic half adder/subtractor, ring resonator, plane wave expansion method, optical Kerr effect, finite-difference time-domain method

Posted Date: May 10th, 2021

DOl: https://doi.org/10.21203/rs.3.rs-475266/v1

License: (c) (1) This work is licensed under a Creative Commons Attribution 4.0 International License.

Read Full License

Version of Record: A version of this preprint was published at Optical and Quantum Electronics on July 4th, 2021. See the published version at https://doi.org/10.1007/s11082-021-03071-y. 


\title{
Ultra-fast tunable optoelectronic half adder/subtractor based on photonic crystal ring resonators covered by graphene nanoshells
}

\author{
SALEH NAGHIZAdE ${ }^{1}$ AND HAMEd SAgHAEI ${ }^{2}$, \\ ${ }^{1}$ Young Researchers and Elite Club, Tabriz Branch, Islamic Azad University, Tabriz, Iran \\ ${ }^{2}$ Department of Electrical Engineering, Shahrekord Branch, Islamic Azad University, Shahrekord, \\ Iran \\ *Corresponding Author: h.saghaei@iaushk.ac.ir
}

\begin{abstract}
This paper reports a new design of a tunable optoelectronic half adder/subtractor. Two photonic crystal $(\mathrm{PhC})$ ring resonators are used to realize the proposed structure. Several silicon rods surrounded by silica rods covered with graphene nanoshells (GNSs) form every $\mathrm{PhC}$ ring resonator. Setting the chemical potential of GNS with an appropriate gate voltage, we can tune the $\mathrm{PhC}$ resonant mode as desired. The plane wave expansion technique is used to study the photonic band structure of the fundamental PC microstructure, and the finitedifference time-domain method is employed in the final design for solving Maxwell's equations to analyze the light propagation inside the structure. We systematically study the effects of physical parameters on the transmission reflection and absorption spectra. By optimizing the geometric dimensions, resonant absorption peaks can be excited at the same time for GNSs. Our numerical results also reveal the maximum time response is about 0.8 ps. The $200 \mu \mathrm{m}^{2}$ area of the proposed half adder/subtractor makes it the building block of every photonic integrated circuit. Also, the design of various fast signal processing systems in optical communication networks is possible due to using tunable GNSs in $\mathrm{PhC}$ ring resonators. This study can introduce the use of two-dimensional materials in the design and implementation of logic circuits.
\end{abstract}

Keywords: Photonic crystal microstructure, nonlinear material, optoelectronic half adder/subtractor, ring resonator, plane wave expansion method, optical Kerr effect, finitedifference time-domain method.

\section{Introduction}

Graphene is one of the most desirable two-dimensional (2D) materials from the ultraviolet to terahertz frequency ranges. It is due to graphene's key characteristics, such as fast optical response, broadband window, high carrier mobility, excellent band structure, and unique mechanical strength and flexibility [1-3]. It also has electro-optic tunability, zero-band-gap (ZBG), and existing free electrons under zero doping conditions. Graphene has metallic properties in the terahertz frequency range $(\mathrm{THz})$, which has made it a popular material among photonic designers. All mentioned features result in this $2 \mathrm{D}$ material becoming a suitable material for fabricating highly tunable optoelectronic and plasmonic devices. In the range from infrared to terahertz, graphene supports a strong plasmonic response, which significantly increases light and graphene interaction. From the ultraviolet (UV) to near-infrared (NIR) wavelength range, monolayer graphene can only absorb a very small fraction of light due to the lack of plasmonic response in this region. It is about $\sim 2.3 \%$ for the visible to NIR range and $\sim 9 \%$ for the UV range [4]. In order to improve the absorption efficiency of graphene, 
researchers studied multiple atomic layers. Undoubtedly, the optimal performance of graphene as a 2D material, such as photon and electron conductivity, will decrease. Therefore, researchers have proposed various methods to enhance the interaction between single-layer graphene and incident light, some of which include providing structures with a wavelengthscale pattern for graphene sublayers. However, they complicate the manufacturing process and cause irreparable damage to single-layer graphene's electronic and photonic properties because the graphene band structure is susceptible to atomic-scale damage and environmental pollutants. Researchers have presented metamaterials based on periodic $\mathrm{Al}$ arrays to increase light absorption efficiency in graphene by plasmon excitation in the UV wavelength range [5]. Unfortunately, a large amount of light energy applied to the structure is lost due to the high absorption of $\mathrm{Al}$ in the structure. A variety of structures based on the periodic dielectric grating array is used to trap light to increase total absorption. However, these efforts are mainly concentrated in the infrared range, and research is needed on other wavelength ranges as well [6]. Therefore, a metamaterial absorber is required to increase the interaction of light with graphene in the ultraviolet wavelength range. Flexible tunability is also crucial to design and investigate optical communication circuits, and this demand is possible by graphene plasmons (GPs). According to the graphene sheet electron flexibility, the generation of certain Fermi energy band levels $\left(E_{f}\right)$ can be obtained in the graphene layer by several methods such as adding substrate and biasing the gate voltage between the substrate and graphene layer. These methods permit us to modify the optical properties of GPs and the operating parameters of graphenebased optoelectronic devices, such as the central resonance wavelength and quality factor. Optoelectronic circuits are appropriate candidates to realize high-speed transmission in data processing systems. One possible solution to design and realize optoelectronic devices is the optical structures based on photonic crystals (PhCs). It is possible to confine and control the propagation of electromagnetic waves inside the desired $\mathrm{PhC}$-based structures due to the available photonic band gap (PBG) in PhCs. So far a variety of all-optical devices such as filters [7-11], modulators [12,13], sensors [14-18], optical fibers [19-26], fundamental logic gates [27-33], encoders [34-36], decoders [37-39], demultiplexers [40-44], PhC fibers [4549], interferometers [50,51], comparators [52-55], adders and subtractors [9,56,57], analog to digital converters [30,58-61] have been designed and investigated using PhCs. Realizing the optical data processing systems can be obtained using computational operators designed based on PhC structures. Serajmohammadi et al. [62] used waveguides and ring resonators (RRs) inside the $2 \mathrm{D}$ hexagonal lattice of the $\mathrm{PhC}$ structure to realize an optical half adder. The proposed structure works according to constructive and destructive interferences of optical waves. The 180-degree phase difference in inputs leads to destructive interference. For this purpose, different lengths of input waveguides in the XOR gate have been chosen. Their proposed structure has a delay time of $4 \mathrm{ps}$ and a footprint of $1056 \mu \mathrm{m}^{2}$. Neisy et al. [63] proposed another optical half-adder consisting of two nonlinear resonant cavities (RCs). These RCs have different resonant modes due to the physical dimensions; therefore, their coupling operations depend on the power intensity. The proposed half adder has a very short time response of 3ps. The main drawback of all studies is that half adders are not tunable. Although few proposed logic gates are tunable based on the applied intensity, this tunability is limited to a special range of optical wavelengths. An ultrafast all-optical half adder with a total footprint of $249.75 \mu \mathrm{m}^{2}$ was proposed by Hosseinzadeh Sani et al. [27]. Their structure contained two 
power regulators, two nonlinear ring resonators, and several waveguides. They used nonlinear rods in resonators; thus, the effective refractive index of the resonator changes as the intensity of incoming light changes. The desired resonance wavelength was tuned by adjusting the linear and nonlinear rods' radii. Their simulation results revealed that the delay time of their designed half adder is about $3.6 \mathrm{ps}$. We designed a high-speed optical half-adder using $\mathrm{PhC}$ in an area of $158 \mu \mathrm{m}^{2}$ [64]. One of our structure's advantages compared to similar studies was the nonuse of high nonlinear dielectric rods. This resulted in no need to increase the input power to divert the incoming light emission to the desired output. The calculations demonstrated that the proposed half-adder has a steady-state time of 0.8 ps due to its small area. Simulations revealed that the minimum transmission of logic 1 and the maximum transmission of logic 0 are $4 \%$ and $75 \%$, respectively. However, the maximum transmission of $75 \%$ for logic 1 is not an ideal value.

This paper proposes a novel design of an optoelectronic half adder/subtractor to overcome the mentioned issues. It is achieved by using graphene nanoshell (GNS) material in two $\mathrm{PhC}$ ring resonators. The maximum delay time is about $3 \mathrm{ps}$ in the proposed structure, and the total footprint is about $303 \mu \mathrm{m}^{2}$. In all studies, the resonant wavelength of $\mathrm{PhC}$ depends on the rod radius or refractive index. In contrast, in this study, the resonant wavelength depends on the applied voltage to graphene. Furthermore, compactness, tunability, and low time response are the key advantages of our proposed half adder. This paper is organized as follows: In Section 2, we introduce the GNS material, PhC ring resonators, and its optical behavior. In Section 3, the optoelectronic design and its functionality will be discussed, and the results of the current study are presented. The paper is closed by the conclusion in Section 4.

\section{Design, model, and methods}

\subsection{Mathematical background}

The graphene sheet's optoelectronic features make it a popular candidate for designing integrated optoelectronic devices. Some of these exciting features are electronic conductivity, high optical transmission efficiency, broad tunability, and mechanical flexibility. In practice, research groups proposed integrating graphene on insulator substrates such as $\mathrm{Si}$ and $\mathrm{SiO}_{2}$. In this work, the GNS with the thickness of $d$ is deposited around the silica rod on the $\mathrm{SiO}_{2}$ substrate. Charge carrier density $\left(n_{\mathrm{c}}\right)$ of a GNS on $\mathrm{SiO}_{2}$ substrate can be tuned by applying the gate voltage $\left(\mathrm{V}_{\mathrm{g}}\right)$ between the substrate and GNS. The charge carrier density is computed as the following equation $[65,66]$

$$
\mathrm{n}_{\mathrm{c}}=\frac{\mathrm{V}_{\mathrm{g}} \varepsilon_{0} \varepsilon_{\mathrm{r}}}{\mathrm{eh}}=\mathrm{V}_{\mathrm{g}} \mathrm{C}
$$

where $\varepsilon_{0}$ and $\varepsilon$ are the air permittivity and substrate's relative permittivity, respectively. e and $\mathrm{h}$ are the electron charge and the substrate height. In Eq.1, the amount of $\varepsilon_{0} \varepsilon_{\mathrm{r}} /$ eh is equal to $\mathrm{C}$, which is called the gate capacitance. The GNS chemical potential $\left(\mu_{\mathrm{c}}\right)$ is calculated as follows $[67,68]$ :

$$
\mu_{c}=\hbar \mathrm{v}_{\mathrm{f}} \sqrt{\pi \mathrm{n}_{\mathrm{c}}}=\hbar \mathrm{v}_{\mathrm{f}} \sqrt{\pi \mathrm{V}_{g} \mathrm{C}}
$$


where, $\hbar$ is the reduced Plank's constant and $v_{\mathrm{f}}$ is the Fermi velocity. The resonance wavelength of the GNS, $\lambda_{0}$, can be derived from the quasi-static analysis method as [69]:

$$
\lambda_{0} \approx \frac{2 \pi \mathrm{ch}}{\mathrm{e}} \sqrt{\frac{\varepsilon_{\mathrm{eff}} \varepsilon_{0} \mathrm{~d} \zeta}{\mathrm{E}_{\mathrm{f}}}}
$$

where $\varepsilon_{\text {eff }}$ is the effective permittivity of the medium surrounding the GNS that is $\varepsilon_{\text {eff }}=\left(\varepsilon_{\mathrm{SiO}_{2}}+\varepsilon_{0}\right) / 2$, and $d$ is the thickness of GNS [70]. The dimensionless constant $\zeta=3.1$ is a fitting parameter. The conductivity of a GNS $(\delta)$ at $\mathrm{T}=300^{\circ} \mathrm{k}$ for infrared to $\mathrm{THz}$ frequencies is approximated using Drude-like expression as follows[10,71]

$$
\delta \approx \frac{\mathrm{ie}^{2} \mathrm{E}_{\mathrm{f}}}{\pi \hbar^{2}\left(\omega+\mathrm{i} \tau^{-1}\right)}
$$

where $\tau$ and $\omega$ are the electron relaxation time and the angular frequency, respectively. Also, when the number of GNS layer $(\mathrm{N})$ is more than one, the conductivity for N-layer GNS will be $\mathrm{N} \delta$. Recently, published papers showed that the finite-difference time-domain (FDTD) method is a comprehensive technique to study the propagation of electromagnetic waves inside compact optical devices such as PhC-based structures. This method solves Maxwell's equation in a tiny volume of the intended $\mathrm{PhC}$ device [72,73]. In this study, the plane wave expansion (PWE) method calculates the PBG region. The FDTD method is also used to solve Maxwell's equation and study the waveguide's light propagation. The following equation gives the optical absorption of graphene [74]

$$
A(\lambda)=\frac{4 \pi c}{\lambda} n(\lambda) k(\lambda) \int_{V}\left|E_{l}\right|^{2} d V
$$

where $\mathrm{c}$ is the speed of light and in free space, $\mathrm{V}$ is the graphene volume, and $E_{1}$ is the local electric field. According to Eq. (5), the light absorption is proportional to the square of local electric field intensity. The UV absorption in graphene is significantly increased at $\lambda=273 \mathrm{~nm}$ due to the large increase in the electric field.

\subsection{Optoelectronic half adder/subtractor}

The fundamental 2D PhC used in this study has a square lattice of dielectric rods. The rod radius and refractive index are $118 \mathrm{~nm}$ and 3.46, respectively. The plane wave expansion (PWE) method has been employed to calculate the photonic band structure. Figure 1 shows the fundamental $\mathrm{PhC}$ has two PBG regions in TM mode where the first one is at $0.29<\mathrm{a} \lambda<0.42$. In this study, the lattice constant is assumed to be $\mathrm{a}=590 \mathrm{~nm}$; therefore, an optical beam with a wavelength of $1404 \mathrm{~nm}<\lambda<2034 \mathrm{~nm}$ cannot propagate in any PhC direction.

By removing several dielectric rods in the fundamental $\mathrm{PhC}$ structure, a waveguide can be created. Accordingly, the wavelengths in the PBG region can be propagated in this waveguide. A typical half adder contains two input ports (labeled as X and Y shown in Fig. 2(a)) and two output ports, called SUM and CARRY. Table 1 represents the truth table of a typical half adder. 
As shown in the figure, when both inputs are inactive (logic 0 is used for OFF state), i.e., no signal is applied to them, or the amplitude of the input signals is less than a threshold value, then both output ports turn OFF. If only one input port is turned on (logic 1 is used for ON mode), SUM is activated, and CARRY is turned off. When both inputs are active, SUM turns off, and CARRY turns on. Figure 2(a) also shows the proposed half subtractor consisting of two input ports (labeled as X and Y), and two output ports (labeled as D and B), where D is used for the difference bit, and B stands for the borrow bit. This logic circuit operates subtracting between the two inputs $\mathrm{X}$ and $\mathrm{Y}$ as $\mathrm{X}-\mathrm{Y}$, and the results of both half adder and half subtractor operators are summarized in Table 1.

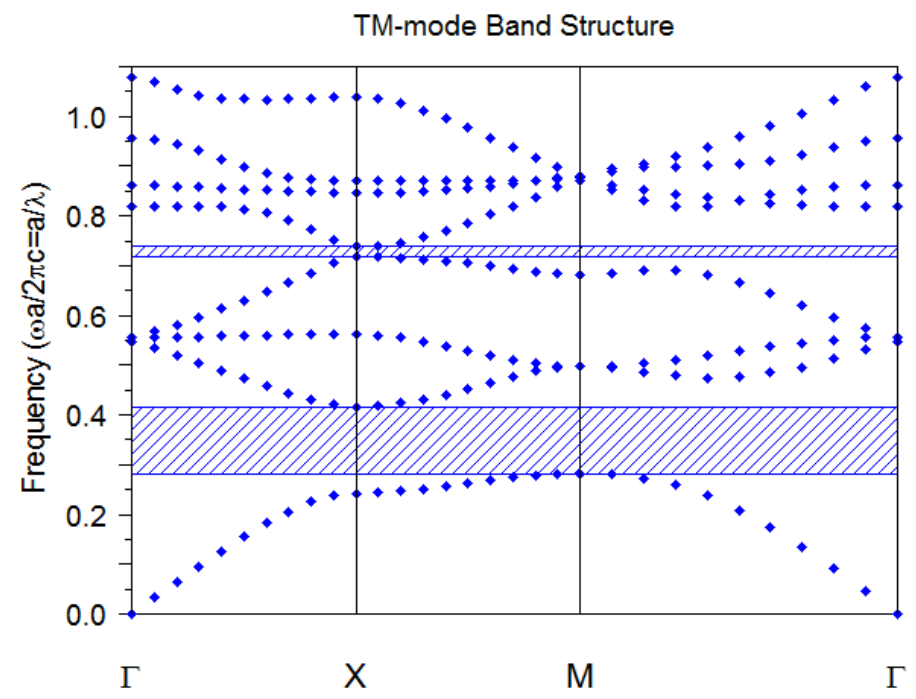

Fig. 1. The band structure diagram of fundamental $\mathrm{PhC}$ in TM mode.

Table 1: Truth table of a conventional half adder/subtractor

\begin{tabular}{|c|c|c|c|c|c|}
\hline \multirow{2}{*}{ Inputs } & \multicolumn{5}{|c|}{ Outputs } \\
\cline { 2 - 6 } & & \multicolumn{2}{|c|}{ Half adder } & \multicolumn{2}{c|}{ Half subtractor } \\
\hline $\mathbf{X}$ & $\mathbf{Y}$ & CARRY & SUM & $\mathbf{D}$ & $\mathbf{B}$ \\
\hline 0 & 0 & 0 & 0 & 0 & 0 \\
\hline 0 & 1 & 0 & 1 & 1 & 0 \\
\hline 1 & 0 & 0 & 1 & 1 & 1 \\
\hline 1 & 1 & 1 & 0 & 0 & 0 \\
\hline
\end{tabular}

To design the proposed operator, we created two input waveguides by removing several dielectric rods called $\mathrm{W} 1$ and $\mathrm{W} 2$. They were connected to form another waveguide labeled as W3. Three dielectric rods with the same radius and smaller than the radius of the rods that make up the structure have the function of light coupling from waveguides W1 and W2 to waveguide W3. Afterward, two output waveguides were made by removing some dielectric rods and called W4 and W5. Finally, two ring resonators were placed between W3 and the output waveguides of W4 and W5. We replaced the outer rods of the ring with defect rods made of silica covered by GNS. The refractive index of silica is assumed to be 1.4. In Fig. 2(a), silicon and silica rods are shown in red and dark green, and GNS shown in dark red surrounds silica rods to tune the resonant mode of light behavior inside the structure depending on the applied gate voltage 
(Vg). The radius of silica rods is $108 \mathrm{~nm}$, and the thickness of GNSs is about $10 \mathrm{~nm}$. As seen in Fig. 2(a), X and Y are used for inputs, and SUM \& D and CARRY \& B are used as output ports. Figure 2(b) shows a 3D perspective view of the proposed structure, where the $\mathrm{SiO}_{2}$ plate shown in gray is the substrate with a thickness of $h=2 \mu \mathrm{m}$. Tuning the output wavelengths is obtained by applying the $\mathrm{Vg}$ to GNSs in every ring resonator. The FDTD method is a numerical method for solving Maxwell's equation in the intended environment and studying electromagnetic wave propagation in $\mathrm{PhC}$-based structures.

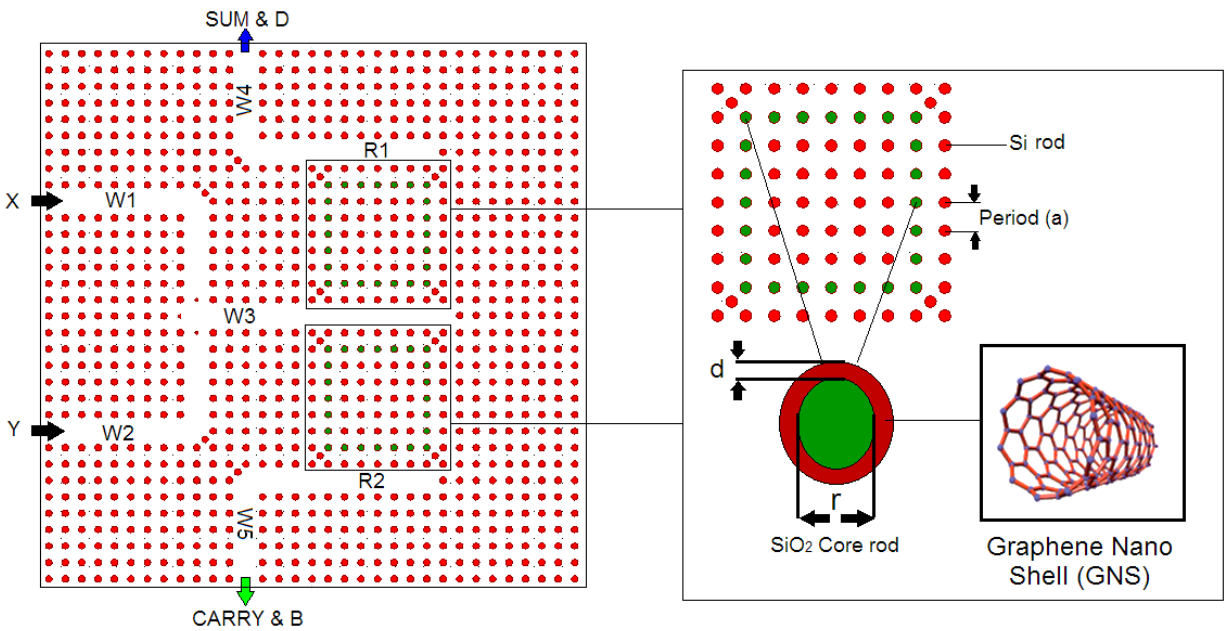

(b)

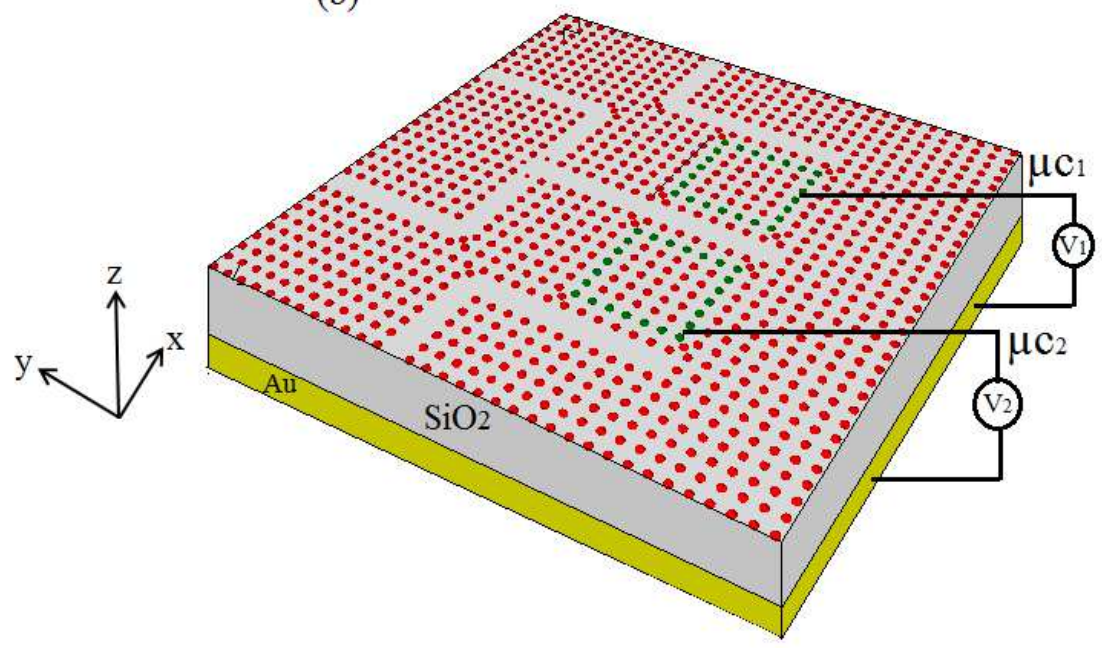

Fig. 2. Illustration of the proposed half adder/subtractor, (a) Top view of XY plane and (b) The perspective view. 


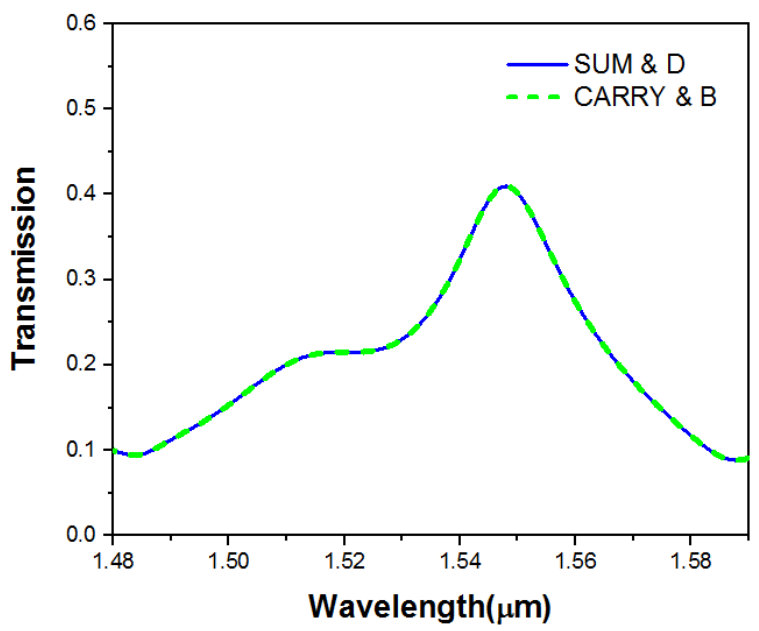

Fig. 3. The ring resonators' output spectra corresponding to SUM \&D and CARRY \& B ports.

\section{Simulation and results}

At first, by choosing the chemical potentials of GNSs with $\mu c_{1}=0.5 \mathrm{ev}$ and $\mu \mathrm{c}_{2}=0.5 \mathrm{ev}$ corresponding to $\mathrm{V}_{1}$ and $\mathrm{V}_{2}$, we aim to study the proposed structure's resonance wavelength at the output ports. Figure 3 shows the ring resonators' output spectra corresponding to SUM \&D and CARRY \& B ports where there is a strong resonant mode at $1550 \mathrm{~nm}$.

In this study, an input optical source centered at $1550 \mathrm{~nm}$ with a pulse width of $3.5 \mathrm{ps}$ is launched into the proposed half adder/subtractor. The gate voltages $(\mathrm{Vg})$ are also applied to GNSs in every ring resonator to control and tune the outputs as desired. For OFF and ON working states, the values of $\mu \mathrm{c}$ set to $0.3 \mathrm{ev}$ and $0.5 \mathrm{ev}$, respectively. Every half adder/subtractor has four different states, which are discussed in the following subsections.

\section{1 Optoelectronic half adder}

Case \#1: When both input ports $\mathrm{X}$ and $\mathrm{Y}$ are inactive, meaning that the signal strength applied to them is negligible, no light beam enters the structure. Therefore, there is no change in the status of the outputs, and the outputs remain in the OFF state.

Case \#2 and Case \#3: when one of the input ports (either X or Y) is ON, due to wavelength matching between the resonance wavelength of the ring resonators and the input optical beam at the appropriate chemical potential of $\mu \mathrm{c}=0.5 \mathrm{ev}$ (It is performed by biasing the $\mathrm{V}_{1}$ ), the first ring resonator will couple the optical beams into W4. Therefore, SUM turns ON, and CARRY remains (See Figs. 4(a) and 4(b)).

Case \#4: Once both input ports are ON (active), we will set the chemical potential $(\mu \mathrm{c})$ of the second ring resonator (R2) to $0.5 \mathrm{ev}$ by biasing $\mathrm{V}_{2}$. Consequently, the first resonator does not couple the optical beam because the $\mathrm{V}_{1}$ is off. Instead, the second resonator transmits light rays to W5. Thus, SUM turns off and CARRY turns on (see Fig. 4 (c))

Table 2 summarizes the simulation results. Comparison of Table 1 with Table 2 reaffirms the designed $\mathrm{PhC}$ structure operates as an optoelectronic half adder. Figure 5 shows the output diagram of the proposed half adder. As shown in Fig. 5(a), when one input port is active (ON state), the output intensities of SUM and CARRY ports are about $85 \%$ and $1 \%$, respectively. 
In this case, the delay time is about 0.8 ps. Figure 5(b) shows, when both input ports are active, the normalized intensities of SUM and CARRY are about $3 \%$ and $170 \%$, respectively. In this case, the delay time is about $0.8 \mathrm{ps}$. It can be seen that the margins of structure for logics 0 $\left(\mathrm{M}_{0}\right)$ and $1\left(\mathrm{M}_{1}\right)$ are equal to $3 \%$ and $85 \%$, respectively. It means that the contrast ratio is 14.5 $\mathrm{dB}$ since this ratio is calculated by $10 \times \log \left(\mathrm{M}_{1} / \mathrm{M}_{0}\right)$. The results show that the proposed structure has a shorter delay time, a greater contrast ratio, and a smaller footprint than previously proposed structures.
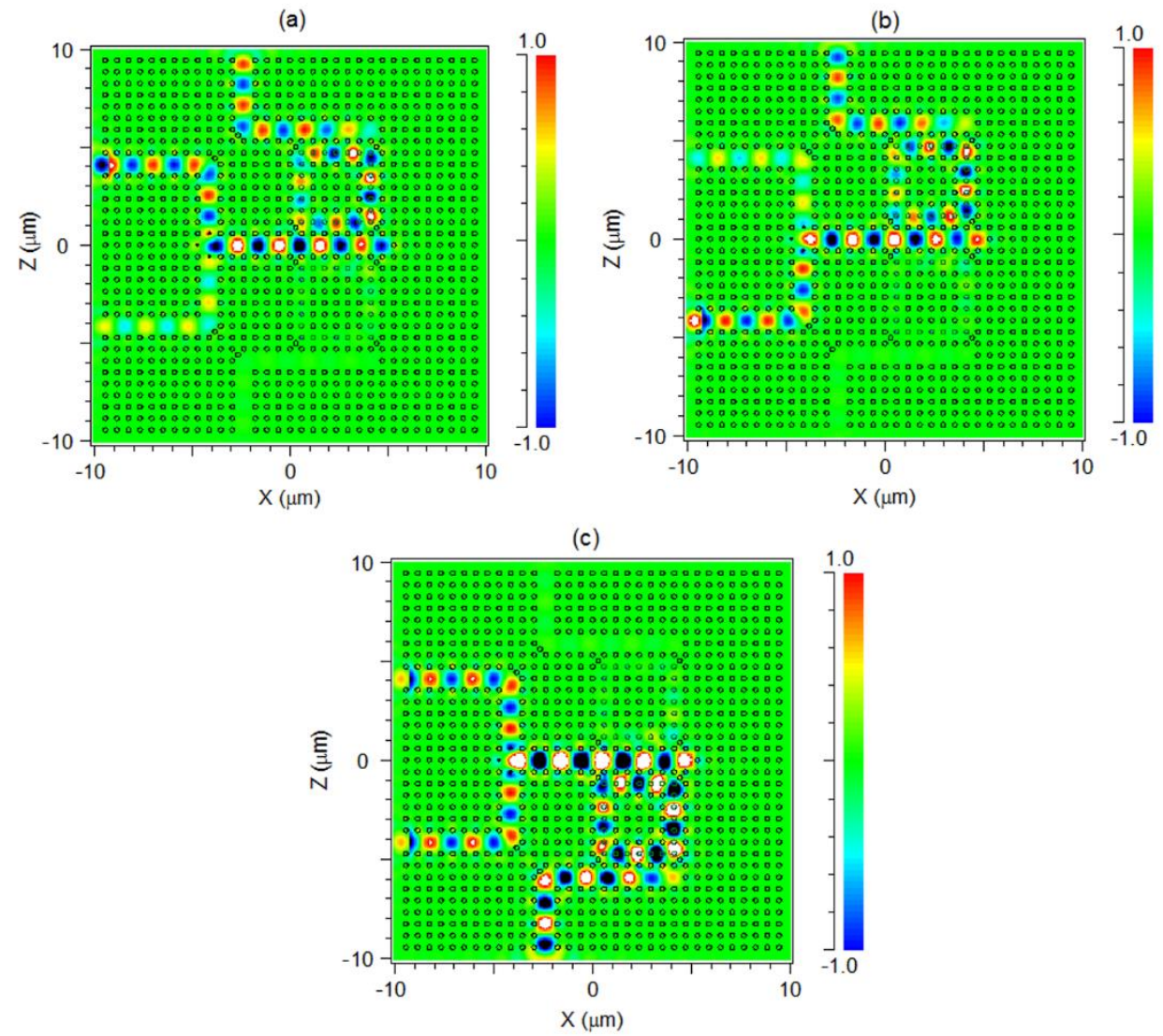

Fig. 4. Light propagation inside half adder for (a) Case $\# 2$ ( $X=1$ and $Y=0)$, (b) Case $\# 3$ ( $X=0$ and $Y=1$ ), and (c) Case \#4 (X=1 and $\mathrm{Y}=1)$.
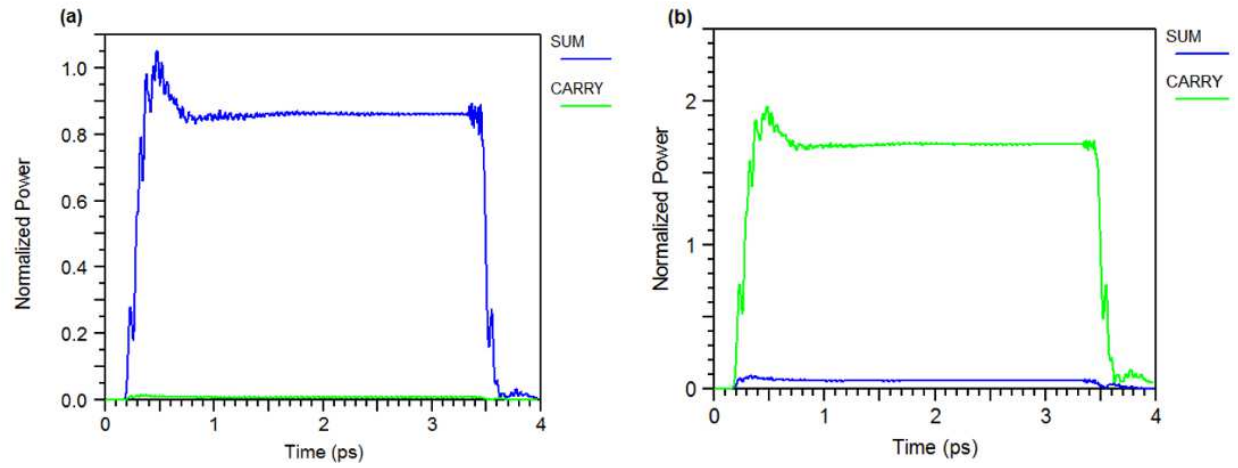

Fig. 5. The proposed half subtractor's time response when (a) one and (b) two input ports are ON. 
Table 2. The truth table for the proposed half adder

\begin{tabular}{cccc}
\hline & & \multicolumn{2}{c}{$\begin{array}{c}\text { Normalized Outputs } \\
\text { I\%) }\end{array}$} \\
\hline Inputs & \multicolumn{3}{c}{ CARR } \\
$\mathrm{X}$ & $\mathrm{Y}$ & $\mathrm{Y}$ & $\mathrm{SUM}$ \\
\hline 0 & 0 & 0 & 0 \\
0 & 1 & 1 & 85 \\
1 & 0 & 1 & 85 \\
1 & 1 & 170 & 3 \\
\hline
\end{tabular}

\section{2 Optoelectronic half subtractor}

Case \#1: When both input ports $\mathrm{X}$ and $\mathrm{Y}$ are inactive (OFF state), no optical beam enters the structure. Therefore, the outputs turn OFF.

Case \#2: When the input port $\mathrm{X}$ is active (ON state), by applying a gate voltage to the GNSs in the first resonator, Wavelength matching between the resonant mode and the input signal occurs at a suitable chemical potential, so the first resonator resonates and transmits light rays to W4. As a result, D turns ON, and B turns OFF (See Fig. 6(a)).

Case \#3: When the input port $\mathrm{Y}$ is active (ON state), by applying gate voltages $\left(\mathrm{V}_{1}\right.$ and $\left.\mathrm{V}_{2}\right)$ to the GNSs in both resonators, wavelength matching occurs between the resonant mode and an input signal for chemical potentials of $\mu \mathrm{c}_{1}=0.5 \mathrm{ev}$ and $\mu \mathrm{c}_{2}=0.5 \mathrm{ev}$, the first and second ring resonators resonate and couple the optical beams into W4 and W5. Therefore, both D and B output ports turn ON. It means the generated code is 11 that is -1 for signed numbers. (See Fig. $6(b))$.

Case \#4: When both input ports are $\mathrm{ON}$, we will set the first and second ring resonators' chemical potentials to $0.3 \mathrm{ev}$ by biasing the $\mathrm{V}_{1}$ and $\mathrm{V}_{2}$. As a result, both resonators cannot couple the optical beam into output ports. Therefore, D and B will be OFF. (See Fig. 6(c)).

Table 2 summarizes the simulation results, and a comparison of Tables 1 and 2 confirms the use of the designed structure as an optoelectronic half subtractor. Figure 7 demonstrates the output diagram of the proposed half adder. As shown in Fig. 7(a), when the first input port is active and the second one is inactive (i.e., $\mathrm{X}=1$ and $\mathrm{Y}=0$ ), the normalized intensities of output ports $\mathrm{D}$ and $\mathrm{B}$ are $45 \%$ and $5 \%$, respectively. In this case, the delay time is about 0.8 ps. Our simulation results in Fig. 7(b)illustrate the normalized intensities of D and B are identical with a value of $45 \%$ when the first input port is inactive and the second one is active (X=0 and $Y=1)$. In this case, the delay time is about $0.8 \mathrm{ps}$. When both input ports are active (X=1 and $Y=1), D$ and B's normalized intensities are less than $3 \%$, and the delay time remains unchanged. The half subtractor margins for logics 0 and 1 are equal to $5 \%$ and $45 \%$, respectively. It means that the contrast ratio is $9.5 \mathrm{~dB}$. The results demonstrate that the proposed structure has a shorter delay time, a greater contrast ratio, and a smaller footprint than previously reported structures. 
Table 3. The truth table for the proposed half subtractor

\begin{tabular}{cccc}
\hline \multicolumn{2}{c}{ Inputs } & \multicolumn{2}{c}{$\begin{array}{c}\text { Normalized Outputs } \\
(\%)\end{array}$} \\
\hline $\mathrm{X}$ & $\mathrm{Y}$ & $\mathrm{B}$ & $\mathrm{D}$ \\
\hline 0 & 0 & 0 & 0 \\
0 & 1 & 1 & 85 \\
1 & 0 & 45 & 45 \\
1 & 1 & 3 & 3 \\
\hline
\end{tabular}

Our numerical results demonstrated that the proposed structure has a short delay time, flexible tunability, and a small footprint. The main advantage of the proposed device is its multipurpose applications compared to previously reported structures so far. It is not sensitive to optical input power variation for switching mechanisms based on the optical beams phase's nonlinear Kerr phenomenon. In addition, our results confirm that by setting an appropriate $\mathrm{Vg}$ to GNS material, this structure can be used as a power splitter, demultiplexer, half adder, and half subtractor.
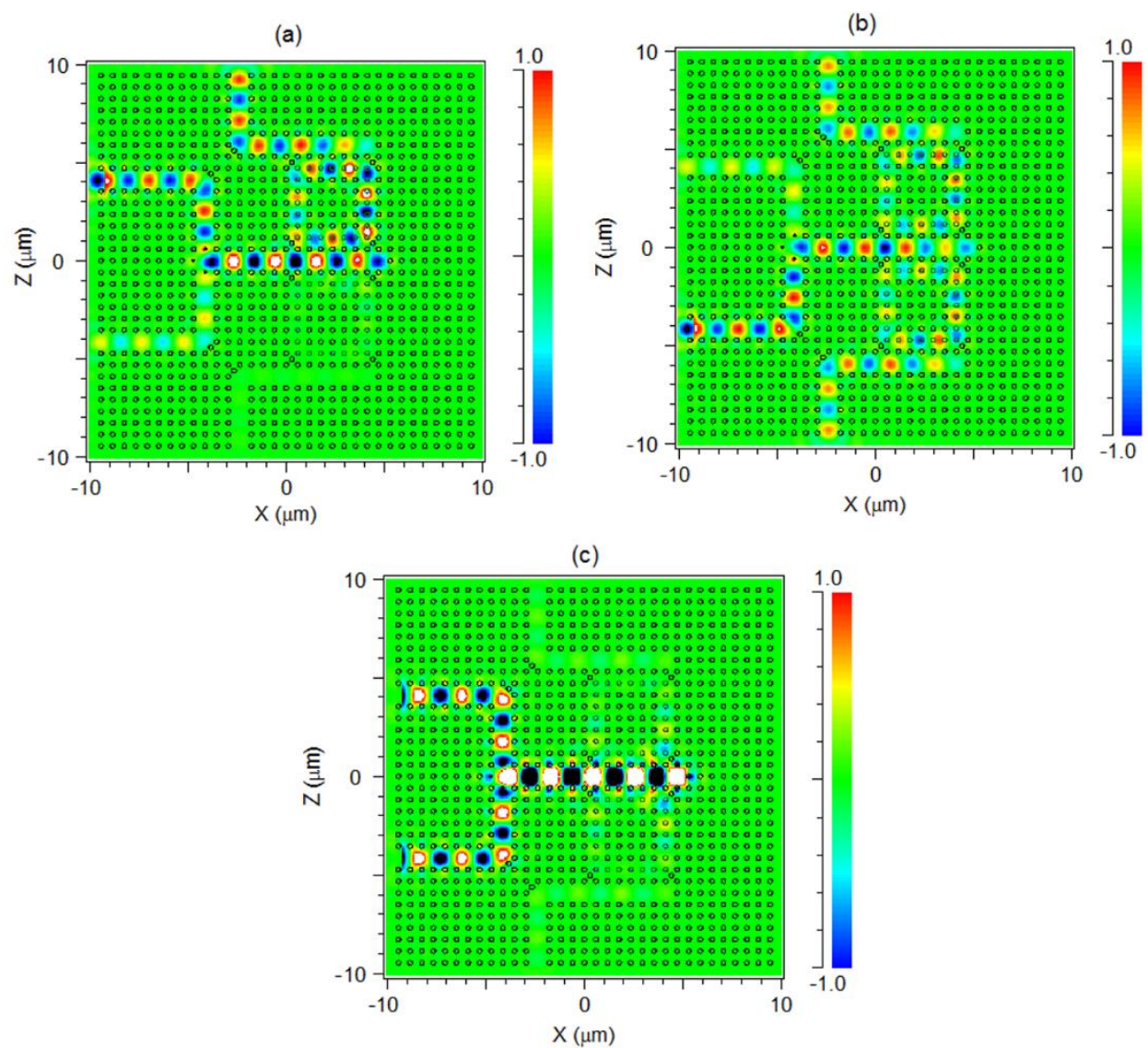

Fig. 6. Light propagation inside half adder for (a) Case $\# 2$ ( $X=1$ and $Y=0$ ), (b) Case \#3 ( $X=0$ and $Y=1$ ), and (c) Case \#4 (X=1 and $\mathrm{Y}=1)$. 

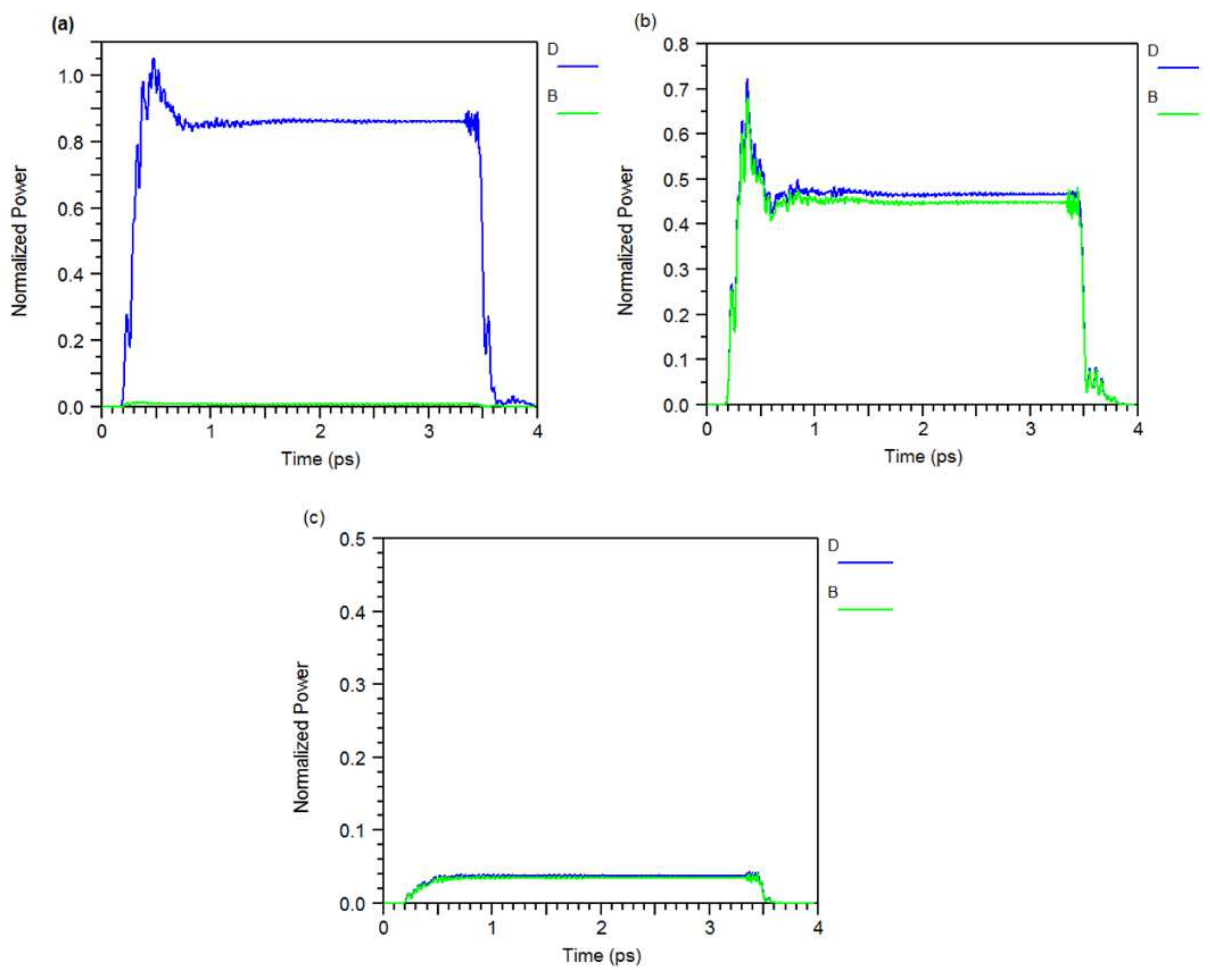

Fig. 7. The time response of the proposed half adder for (a) Case \#2 ( $X=1$ and $Y=0)$, (b) Case \#3 ( $X=0$ and $\mathrm{Y}=1)$, and (c) Case \#4 (X=1 and $\mathrm{Y}=1)$.

\section{Conclusion}

In summary, we presented a new PhC-based structure for realizing all-optical half adder and half subtractor in a single structure. Five waveguides and two $\mathrm{PhC}$ ring resonators formed the final structure. Every $\mathrm{PhC}$ ring resonator consisted of several silicon rods surrounded by silica rods covered by GNSs. Our results revealed that the proposed structure's total footprint and maximum delay time are about $379 \mu \mathrm{m}^{2}$ and $0.8 \mathrm{ps}$, respectively. Compared to other recently published papers, the proposed structure's main advantage is its tunability by changing graphene's chemical potential. Graphene-based logic gates open a new window to develop and fabricate the multipurpose optoelectronic circuits for signal processing systems applications.

\section{Funding}

This research received no specific grant from any funding agency in the public, commercial, or not-for-profit sectors.

\section{Disclosures}

The authors declare no conflicts of interest.

\section{Author contributions}

Saleh Naghizade: Conceptualization, Data curation, Formal analysis, Funding acquisition, Investigation, Methodology, Software. Hamed Saghaei: Project administration, Resources, Software, Supervision, Validation, Visualization, Roles/Writing - original draft, Writing review \& editing. 


\section{Availability of data and material}

The data that support the findings of this study are available from the corresponding author upon reasonable request.

\section{Compliance with ethical standards}

The authors have completely observed the ethical issues, including plagiarism, informed consent, misconduct, data fabrication and/or falsification, double publication and/or submission, redundancy.

\section{References}

1. Z. Sun, A. Martinez, en F. Wang, "Optical modulators with 2D layered materials," Nat. Photonics 10(4), 227-238 (2016).

2. R. Tang, S. Han, F. Teng, K. Hu, Z. Zhang, M. Hu, en X. Fang, "Size-Controlled Graphene Nanodot Arrays/ZnO Hybrids for High-Performance UV Photodetectors," Adv. Sci. 5(1), 1700334 (2018).

3. L. Ye, Y. Chen, G. Cai, N. Liu, J. Zhu, Z. Song, en Q. H. Liu, "Broadband absorber with periodically sinusoidally-patterned graphene layer in terahertz range," Opt. Express 25(10), 11223-11232 (2017).

4. R. R. Nair, P. Blake, A. N. Grigorenko, K. S. Novoselov, T. J. Booth, T. Stauber, N. M. R. Peres, en A. K. Geim, "Fine structure constant defines visual transparency of graphene," Science (80-. ). 320(5881), 1308 (2008).

5. R. González-Campuzano, J. M. Saniger, en D. Mendoza, "Plasmonic resonances in hybrid systems of aluminum nanostructured arrays and few layer graphene within the UV-IR spectral range," Nanotechnology 28(46), 465704 (2017).

6. C. Guo, Z. Zhu, X. Yuan, W. Ye, K. Liu, J. Zhang, W. Xu, en S. Qin, "Experimental demonstration of total absorption over $99 \%$ in the near infrared for monolayer-graphene-based subwavelength structures," Adv. Opt. Mater. 4(12), 1955-1960 (2016).

7. H. Butt, Q. Dai, P. Farah, T. Butler, T. D. Wilkinson, J. J. Baumberg, en G. A. J. Amaratunga, "Metamaterial high pass filter based on periodic wire arrays of multiwalled carbon nanotubes," Appl. Phys. Lett. 97(16), 163102 (2010).

8. I. J. H. Mccrindle, J. Grant, T. D. Drysdale, en D. R. S. Cumming, "Multi-spectral materials: Hybridisation of optical plasmonic filters and a terahertz metamaterial absorber," Adv. Opt. Mater. 2(2), 149-153 (2014).

9. M. Hosseinzadeh Sani, A. Ghanbari, en H. Saghaei, "An ultra-narrowband all-optical filter based on the resonant cavities in rod-based photonic crystal microstructure," Opt. Quantum Electron. 52(6), 295 (2020).

10. S. Naghizade en H. Saghaei, "Tunable graphene-on-insulator band-stop filter at the mid-infrared region," Opt. Quantum Electron. 52(4), 224 (2020).

11. F. Tavakoli, F. B. Zarrabi, en H. Saghaei, "Modeling and analysis of high-sensitivity refractive index sensors based on plasmonic absorbers with Fano response in the near-infrared spectral region," Appl. Opt. 58(20), 5404-5414 (2019).

12. D. Shrekenhamer, J. Montoya, S. Krishna, en W. J. Padilla, "Four-color metamaterial absorber THz spatial light modulator," Adv. Opt. Mater. 1(12), 905-909 (2013).

13. C. M. Watts, D. Shrekenhamer, J. Montoya, G. Lipworth, J. Hunt, T. Sleasman, S. Krishna, D. R. Smith, en W. J. Padilla, "Terahertz compressive imaging with metamaterial spatial light modulators," Nat. Photonics 8(8), 605-609 (2014).

14. B. X. Wang, X. Zhai, G. Z. Wang, W. Q. Huang, en L. L. Wang, "A novel dual-band terahertz metamaterial absorber for a sensor application," J. Appl. Phys. 117(1), 14504 (2015).

15. R. Yahiaoui, S. Tan, L. Cong, R. Singh, F. Yan, en W. Zhang, "Multispectral terahertz sensing with highly flexible ultrathin metamaterial absorber," J. Appl. Phys. 118(8), 83103 (2015).

16. M. Hosseinzadeh Sani, H. Saghaei, M. A. Mehranpour, en A. Asgariyan Tabrizi, "A novel all-optical sensor design based on a tunable resonant nanocavity in photonic crystal microstructure applicable in MEMS accelerometers," Photonic Sensors (2020).

17. A. Farmani, "Three-dimensional FDTD analysis of a nanostructured plasmonic sensor in the near-infrared 
range," JOSA B 36(2), 401-407 (2019).

18. A. Farmani en A. Mir, "Graphene sensor based on surface plasmon resonance for optical scanning," IEEE Photonics Technol. Lett. 31(8), 643-646 (2019).

19. A. Ghanbari, A. Kashaninia, A. Sadr, en H. Saghaei, "Supercontinuum generation with femtosecond optical pulse compression in silicon photonic crystal fibers at $2500 \mathrm{~nm}$," Opt. Quantum Electron. 50(11), (2018).

20. H. Saghaei, V. Heidari, M. Ebnali-Heidari, en M. R. Yazdani, "A systematic study of linear and nonlinear properties of photonic crystal fibers," Optik (Stuttg). 127(24), 11938-11947 (2016).

21. H. Saghaei, M. Ebnali-Heidari, en M. K. Moravvej-Farshi, "Midinfrared supercontinuum generation via As_2Se_3 chalcogenide photonic crystal fibers," Appl. Opt. 54(8), 2072 (2015).

22. H. Saghaei, M. K. Moravvej-Farshi, M. Ebnali-Heidari, en M. N. Moghadasi, "Ultra-Wide Mid-Infrared Supercontinuum Generation in As40Se60 Chalcogenide Fibers: Solid Core PCF Versus SIF," IEEE J. Sel. Top. Quantum Electron. 22(2), (2016).

23. M. Aliee, M. H. Mozaffari, en H. Saghaei, "Dispersion-flattened photonic quasicrystal optofluidic fiber for telecom C band operation," Photonics Nanostructures - Fundam. Appl. 40, 100797 (2020).

24. A. Ghanbari, A. Kashaninia, A. Sadr, en H. Saghaei, "Supercontinuum generation for optical coherence tomography using magnesium fluoride photonic crystal fiber," Optik (Stuttg). 140, 545-554 (2017).

25. M. Diouf, A. Ben Salem, R. Cherif, H. Saghaei, en A. Wague, "Super-flat coherent supercontinuum source in As_388Se_612 chalcogenide photonic crystal fiber with all-normal dispersion engineering at a very low input energy," Appl. Opt. 56(2), 163 (2017).

26. H. Saghaei, "Supercontinuum source for dense wavelength division multiplexing in square photonic crystal fiber via fluidic infiltration approach," Radioengineering 26(1), 16-22 (2017).

27. M. H. Sani, A. A. Tabrizi, H. Saghaei, en R. Karimzadeh, "An ultrafast all-optical half adder using nonlinear ring resonators in photonic crystal microstructure," Opt. Quantum Electron. 52(2), 107 (2020).

28. S. Naghizade en H. Saghaei, "A novel design of all-optical 4 to 2 encoder with multiple defects in silicabased photonic crystal fiber," Optik (Stuttg). 222, 165419 (2020).

29. T. A. Moniem, "All-optical digital $4 \times 2$ encoder based on 2D photonic crystal ring resonators," J. Mod. Opt. 63(8), 735-741 (2016).

30. F. Mehdizadeh, M. Soroosh, H. Alipour-Banaei, en E. Farshidi, "All optical 2-bit analog to digital converter using photonic crystal based cavities," Opt. Quantum Electron. 49(1), 38 (2017).

31. H. Saghaei, A. Zahedi, R. Karimzadeh, en F. Parandin, "Line defects on photonic crystals for the design of all-optical power splitters and digital logic gates," Superlattices Microstruct. 110, 133-138 (2017).

32. M. Danaie en H. Kaatuzian, "Design and simulation of an all-optical photonic crystal AND gate using nonlinear Kerr effect," Opt. Quantum Electron. 44(1), 27-34 (2012).

33. M. Moradi, M. Danaie, en A. A. Orouji, "Design of all-optical XOR and XNOR logic gates based on Fano resonance in plasmonic ring resonators," Opt. Quantum Electron. 51(5), 154 (2019).

34. F. Mehdizadeh, M. Soroosh, en H. Alipour-Banaei, "Proposal for 4-to-2 optical encoder based on photonic crystals," IET Optoelectron. 11(1), 29-35 (2017).

35. S. Naghizade en H. Khoshsima, "Low input power an all optical $4 \times 2$ encoder based on triangular lattice shape photonic crystal," J. Opt. Commun. 1, 1-8 (2018).

36. F. Haddadan, M. Soroosh, en N. Alaei-Sheini, "Designing an electro-optical encoder based on photonic crystals using the graphene-Al 2 O 3 stacks," Appl. Opt. 59(7), 2179-2185 (2020).

37. H. Alipour-Banaei, F. Mehdizadeh, S. Serajmohammadi, en M. Hassangholizadeh-Kashtiban, "A $2 * 4$ all optical decoder switch based on photonic crystal ring resonators," J. Mod. Opt. 62(6), 430-434 (2015).

38. F. Parandin, M. M. Karkhanehchi, M. Naseri, en A. Zahedi, "Design of a high bitrate optical decoder based on photonic crystals," J. Comput. Electron. 17(2), 830-836 (2018).

39. S. Salimzadeh en H. Alipour-Banaei, "A novel proposal for all optical 3 to 8 decoder based on nonlinear ring resonators," J. Mod. Opt. 65(17), 2017-2024 (2018).

40. F. Mehdizadeh en M. Soroosh, "A new proposal for eight-channel optical demultiplexer based on photonic crystal resonant cavities," Photonic Netw. Commun. 31(1), 65-70 (2016).

41. R. Talebzadeh, M. Soroosh, Y. S. Kavian, en F. Mehdizadeh, "Eight-channel all-optical demultiplexer based on photonic crystal resonant cavities," Optik (Stuttg). 140, 331-337 (2017).

42. K. Wen, L. Yan, W. Pan, B. Luo, Z. Guo, en Y. Guo, "Wavelength demultiplexing structure based on a plasmonic metal-insulator-metal waveguide," J. Opt. (United Kingdom) 14(7), 75001 (2012).

43. H. Saghaei, B. Seyfe, H. Bakhshi, en R. Bayat, "Novel approach to adjust the step size for closed-loop power control in wireless cellular code division multiple access systems under flat fading," IET Commun. 5(11), 1469-1483 (2011).

44. H. Saghaei en B. Seyfe, "New approach to closed-loop power control in cellular CDMA systems under multipath fading," in 2008 International Conference on Wireless Communications, Networking and Mobile Computing, WiCOM 2008 (2008). 
45. H. Saghaei, "Dispersion-engineered microstructured optical fiber for mid-infrared supercontinuum generation," Appl. Opt. 57(20), 5591 (2018).

46. M. Ebnali-Heidari, H. Saghaei, F. Koohi-Kamali, M. Naser Moghadasi, en M. K. Moravvej-Farshi, "Proposal for Supercontinuum Generation by Optofluidic Infiltrated Photonic Crystal Fibers," IEEE J. Sel. Top. Quantum Electron. 20(5), (2014).

47. R. Raei, M. Ebnali-Heidari, en H. Saghaei, "Supercontinuum generation in organic liquid-liquid corecladding photonic crystal fiber in visible and near-infrared regions: publisher's note," J. Opt. Soc. Am. B 35(7), 1545 (2018).

48. H. Saghaei en V. Van, "Broadband mid-infrared supercontinuum generation in dispersion-engineered silicon-on-insulator waveguide," J. Opt. Soc. Am. B 36(2), A193 (2019).

49. H. Saghaei en A. Ghanbari, "White light generation using photonic crystal fiber with sub-micron circular lattice," J. Electr. Eng. 68(4), 282-289 (2017).

50. L. Gu, W. Jiang, X. Chen, L. Wang, en R. T. Chen, "High speed silicon photonic crystal waveguide modulator for low voltage operation," Appl. Phys. Lett. 90(7), 71105 (2007).

51. H. Saghaei, P. Elyasi, en R. Karimzadeh, "Design, fabrication, and characterization of Mach-Zehnder interferometers," Photonics Nanostructures - Fundam. Appl. 37, 100733 (2019).

52. V. Fakouri-Farid en A. Andalib, "Design and simulation of an all optical photonic crystal-based comparator," Optik (Stuttg). 172, 241-248 (2018).

53. H. Jile, "Realization of an all-optical comparator using beam interference inside photonic crystal waveguides," Appl. Opt. 59(12), 3714 (2020).

54. M. Danaie en H. Kaatuzian, "Design of a photonic crystal differential phase comparator for a MachZehnder switch," J. Opt. 13(1), 15504 (2010).

55. M. Danaie en H. Kaatuzian, "Bandwidth Improvement for a Photonic Crystal Optical Y-splitter," J. Opt. Soc. Korea 15(3), 283-288 (2011).

56. R. Moradi, "All optical half subtractor using photonic crystal based nonlinear ring resonators," Opt. Quantum Electron. 51(4), 119 (2019).

57. M. Moradi, M. Danaie, en A. A. Orouji, "Design and analysis of an optical full-adder based on nonlinear photonic crystal ring resonators," Optik (Stuttg). 172, 127-136 (2018).

58. A. Rostami en G. Rostami, "Full optical analog to digital (A/D) converter based on Kerr-like nonlinear ring resonator," Opt. Commun. 228(1-3), 39-48 (2003).

59. A. Tavousi, M. A. Mansouri-Birjandi, en M. Saffari, "Successive approximation-like 4-bit full-optical analog-to-digital converter based on Kerr-like nonlinear photonic crystal ring resonators," Phys. E LowDimensional Syst. Nanostructures 83, 101-106 (2016).

60. K. Fasihi, "All-optical analog-to-digital converters based on cascaded 3-dB power splitters in 2D photonic crystals," Optik (Stuttg). 125(21), 6520-6523 (2014).

61. B. Youssefi, M. K. Moravvej-Farshi, en N. Granpayeh, "Two bit all-optical analog-to-digital converter based on nonlinear Kerr effect in 2D photonic crystals," Opt. Commun. 285(13-14), 3228-3233 (2012).

62. S. Serajmohammadi, H. Alipour-Banaei, en F. Mehdizadeh, "Proposal for realizing an all-optical half adder based on photonic crystals," Appl. Opt. 57(7), 1617-1621 (2018).

63. M. Neisy, M. Soroosh, en K. Ansari-Asl, "All optical half adder based on photonic crystal resonant cavities," Photonic Netw. Commun. 35(2), 245-250 (2018).

64. S. Naghizade en H. Saghaei, "A Novel Design of All-Optical Half-Adder Using a Linear Defect in Photonic Crystal Microstructure," J. Appl. Res. Electr. Eng. (2020).

65. T. T. M. Thi, P. T. Dat, V. C. Minh, N. H. Tho, en N. X. Sang, "Physical Adsorption and Photocatalytic Activity of Titanium Dioxide Nanotube and Graphene Oxide Composite," VNU J. Sci. Nat. Sci. Technol. 34(3), (2018).

66. B. Shi, W. Cai, X. Zhang, Y. Xiang, Y. Zhan, J. Geng, M. Ren, en J. Xu, "Tunable band-stop filters for graphene plasmons based on periodically modulated graphene," Sci. Rep. 6(1), 1-7 (2016).

67. L. Ju, B. Geng, J. Horng, C. Girit, M. Martin, Z. Hao, H. A. Bechtel, X. Liang, A. Zettl, Y. R. Shen, en F. Wang, "Graphene plasmonics for tunable terahertz metamaterials," Nat. Nanotechnol. 6(10), 630-634 (2011).

68. A. Farmani, M. Miri, en M. H. Sheikhi, "Analytical modeling of highly tunable giant lateral shift in total reflection of light beams from a graphene containing structure," Opt. Commun. 391, 68-76 (2017).

69. H. Yan, X. Li, B. Chandra, G. Tulevski, Y. Wu, M. Freitag, W. Zhu, P. Avouris, en F. Xia, "Tunable infrared plasmonic devices using graphene/insulator stacks," Nat. Nanotechnol. 7(5), 330-334 (2012).

70. A. Farmani, M. Miri, en M. H. Sheikhi, "Design of a high extinction ratio tunable graphene on white graphene polarizer," IEEE Photonics Technol. Lett. 30(2), 153-156 (2017).

71. C. Casiraghi, A. Hartschuh, E. Lidorikis, H. Qian, H. Harutyunyan, T. Gokus, K. S. Novoselov, en A. C. Ferrari, "Rayleigh imaging of graphene and graphene layers," Nano Lett. 7(9), 2711-2717 (2007).

72. S. Johnson en J. Joannopoulos, "Block-iterative frequency-domain methods for Maxwell's equations in a 
planewave basis," Opt. Express 8(3), 173 (2001).

73. S. D. Gedney, "Introduction to the Finite-Difference Time-Domain (FDTD) method for electromagnetics," Synth. Lect. Comput. Electromagn. 27(1), 1-250 (2011).

74. H. Farmani, A. Farmani, en Z. Biglari, "A label-free graphene-based nanosensor using surface plasmon resonance for biomaterials detection," Phys. E Low-dimensional Syst. Nanostructures 116, 113730 (2020). 
Figures

TM-mode Band Structure

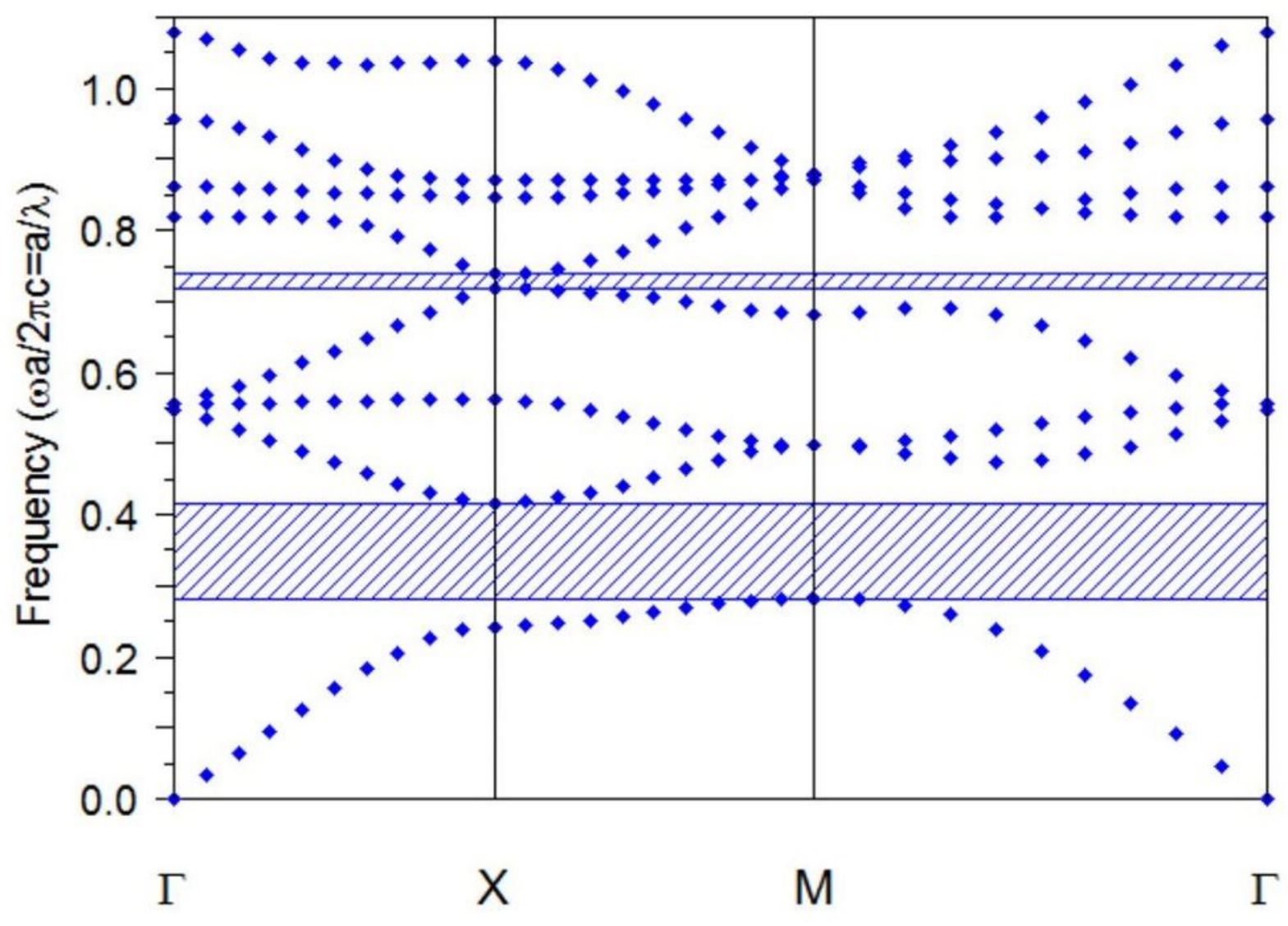

Figure 1

The band structure diagram of fundamental $\mathrm{PhC}$ in TM mode. 


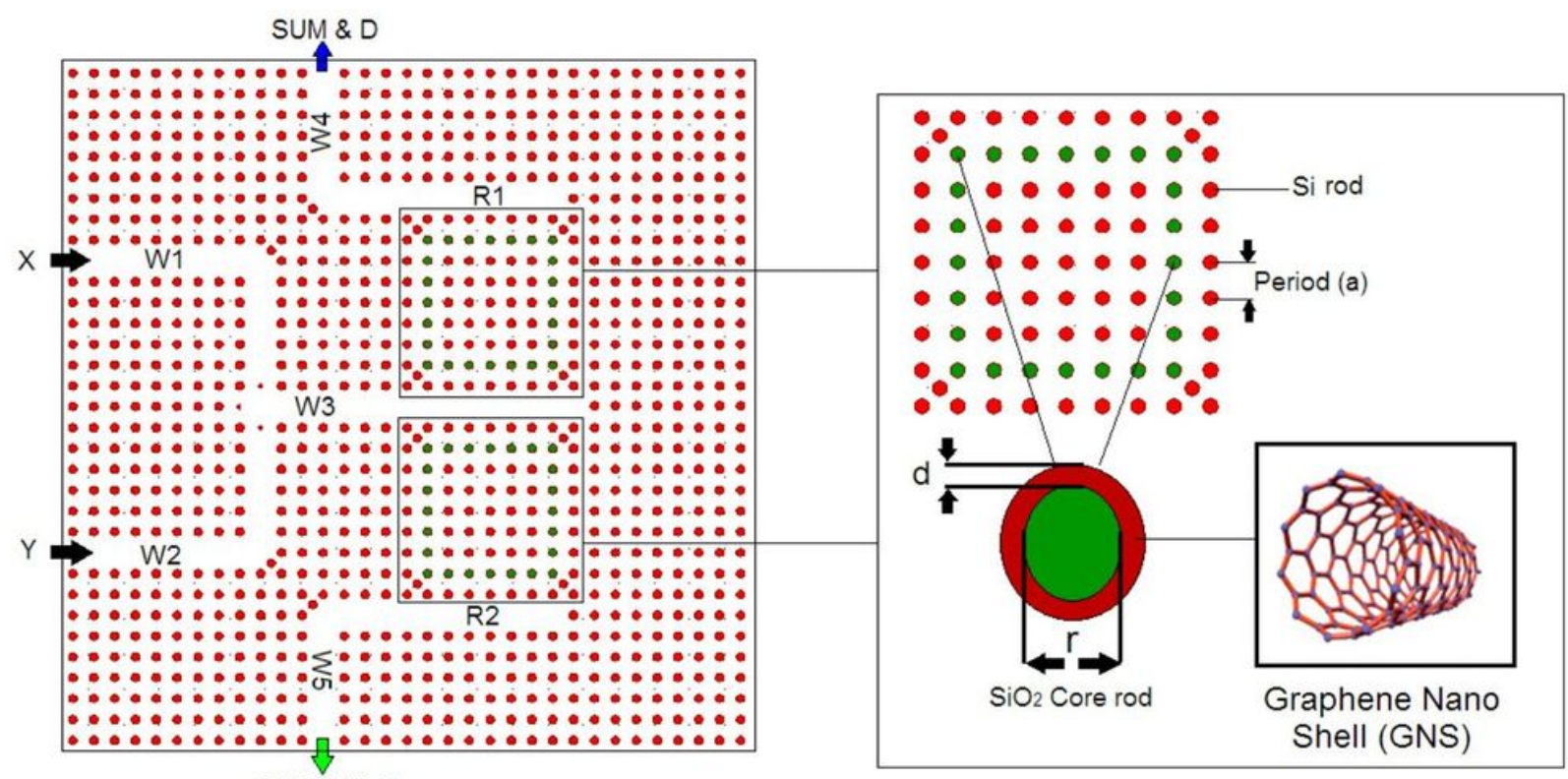

CARRY \& B

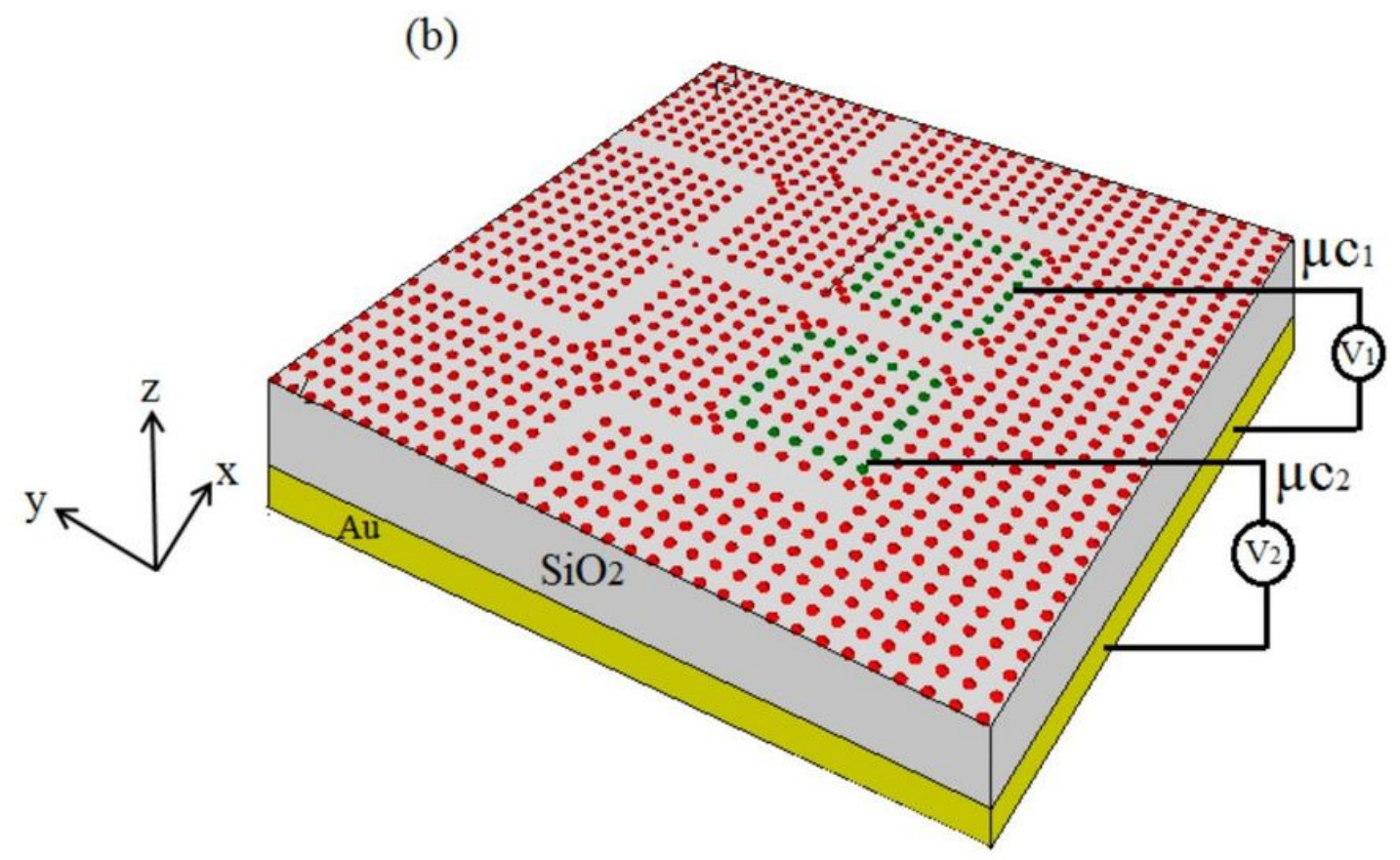

Figure 2

Illustration of the proposed half adder/subtractor, (a) Top view of XY plane and (b) The perspective view. 


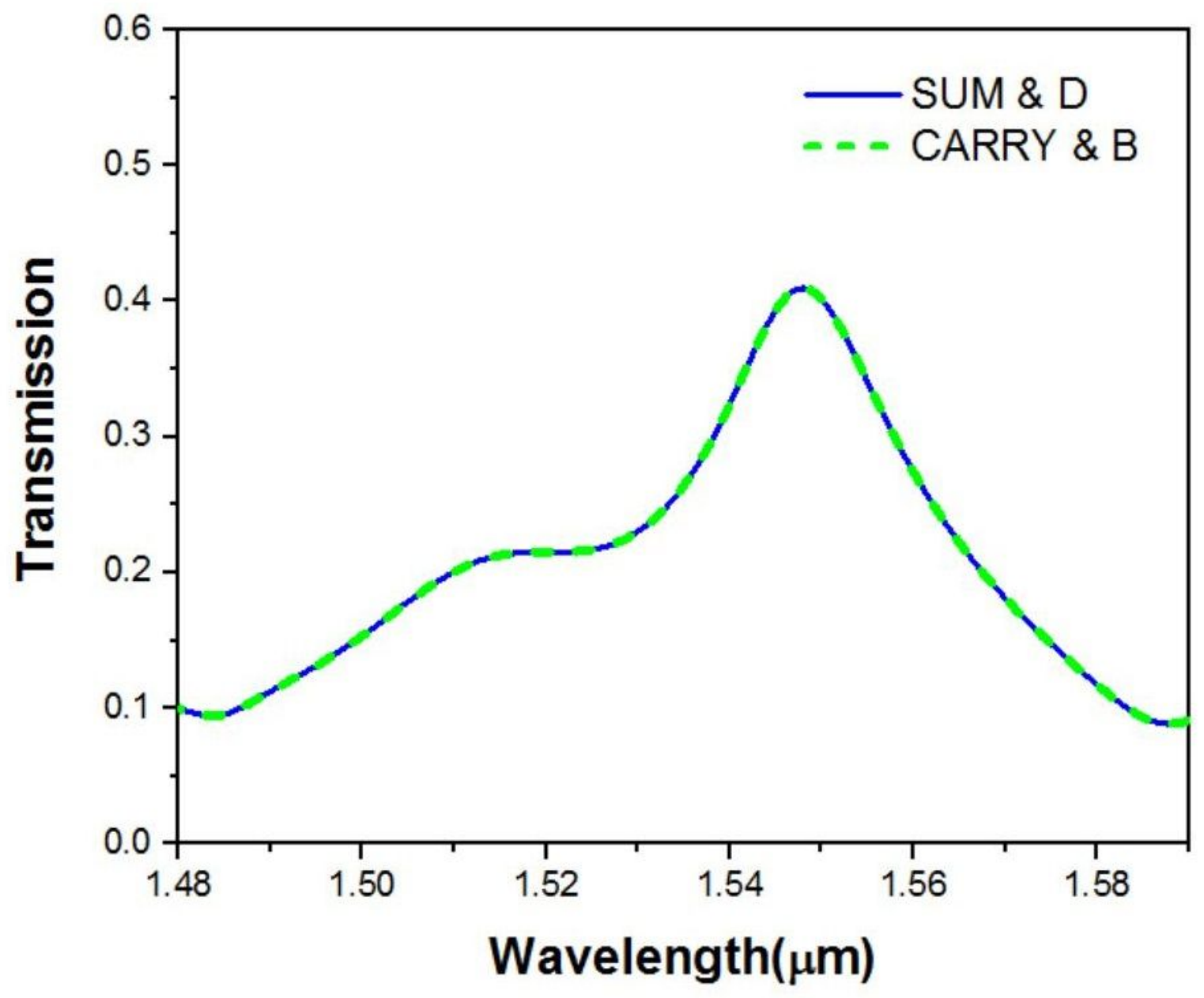

Figure 3

The ring resonators' output spectra corresponding to SUM \&D and CARRY \& B ports. 
(a)

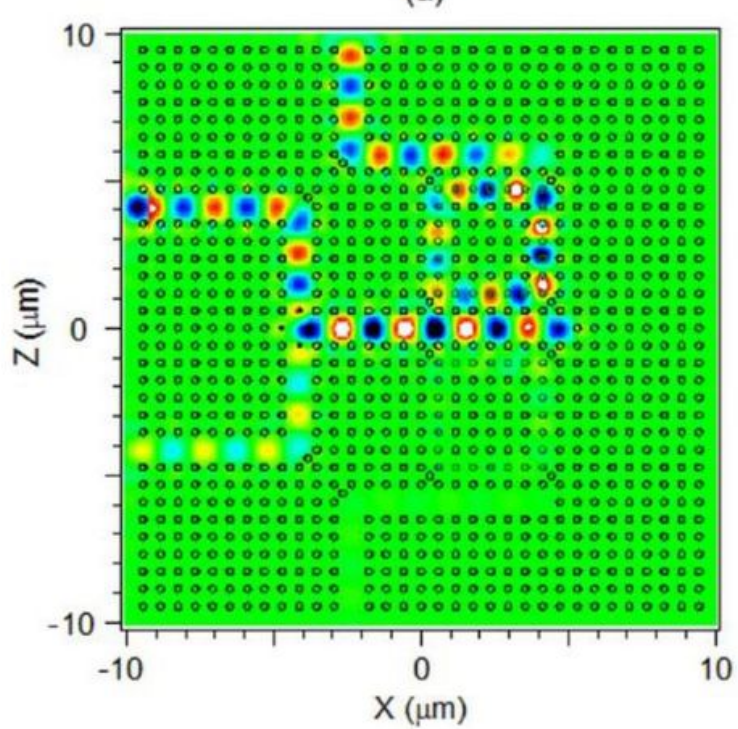

(b)

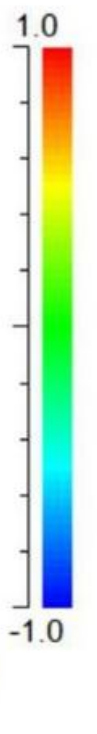

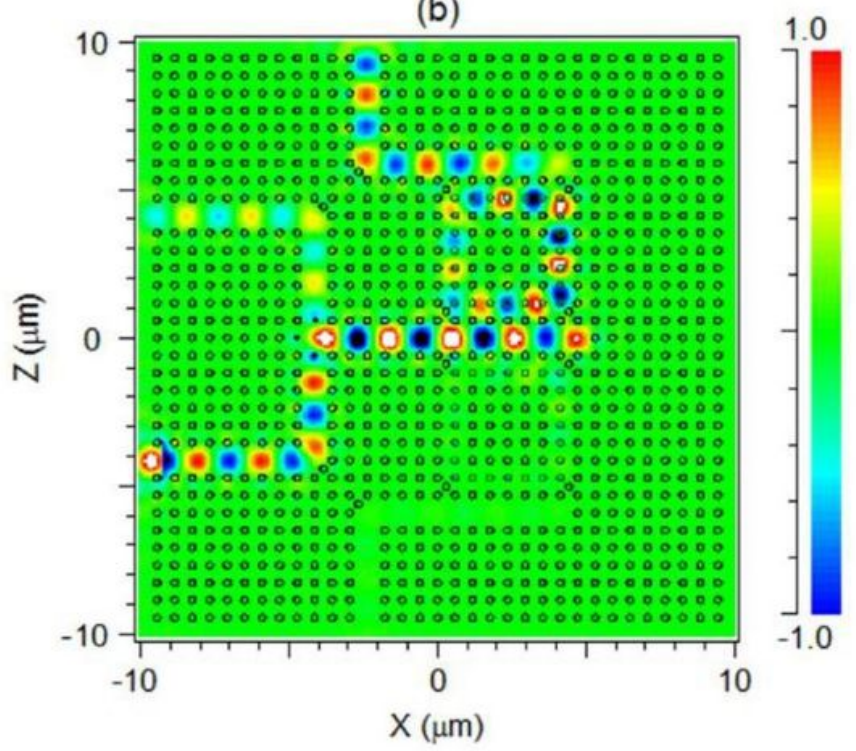

(c)

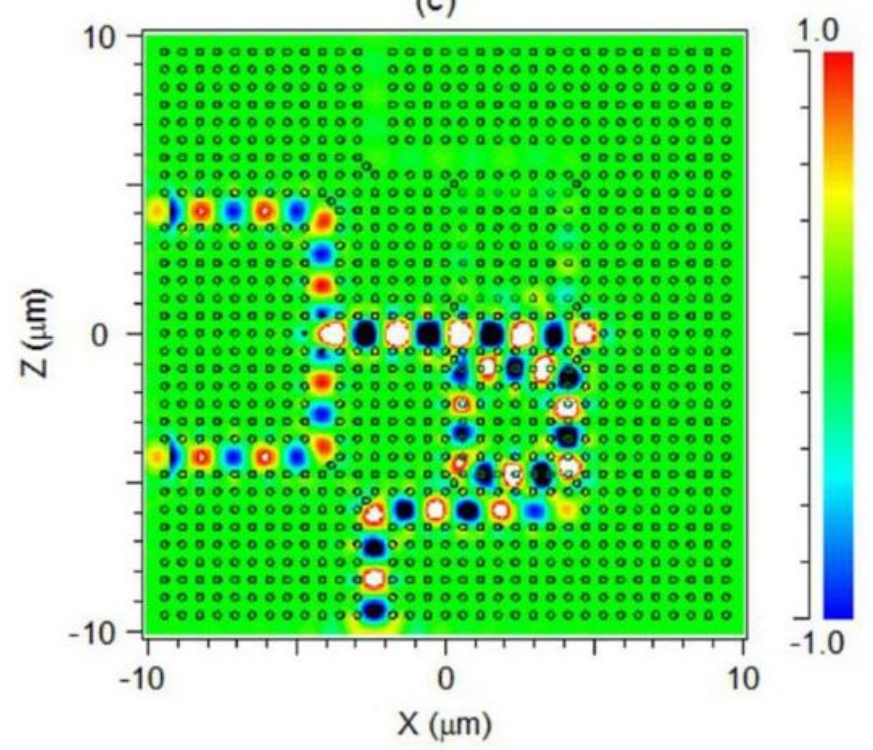

\section{Figure 4}

Light propagation inside half adder for (a) Case \#2 ( $X=1$ and $Y=0)$, (b) Case \#3 ( $X=0$ and $Y=1)$, and (c) Case \#4 ( $\mathrm{X}=1$ and $\mathrm{Y}=1)$. 

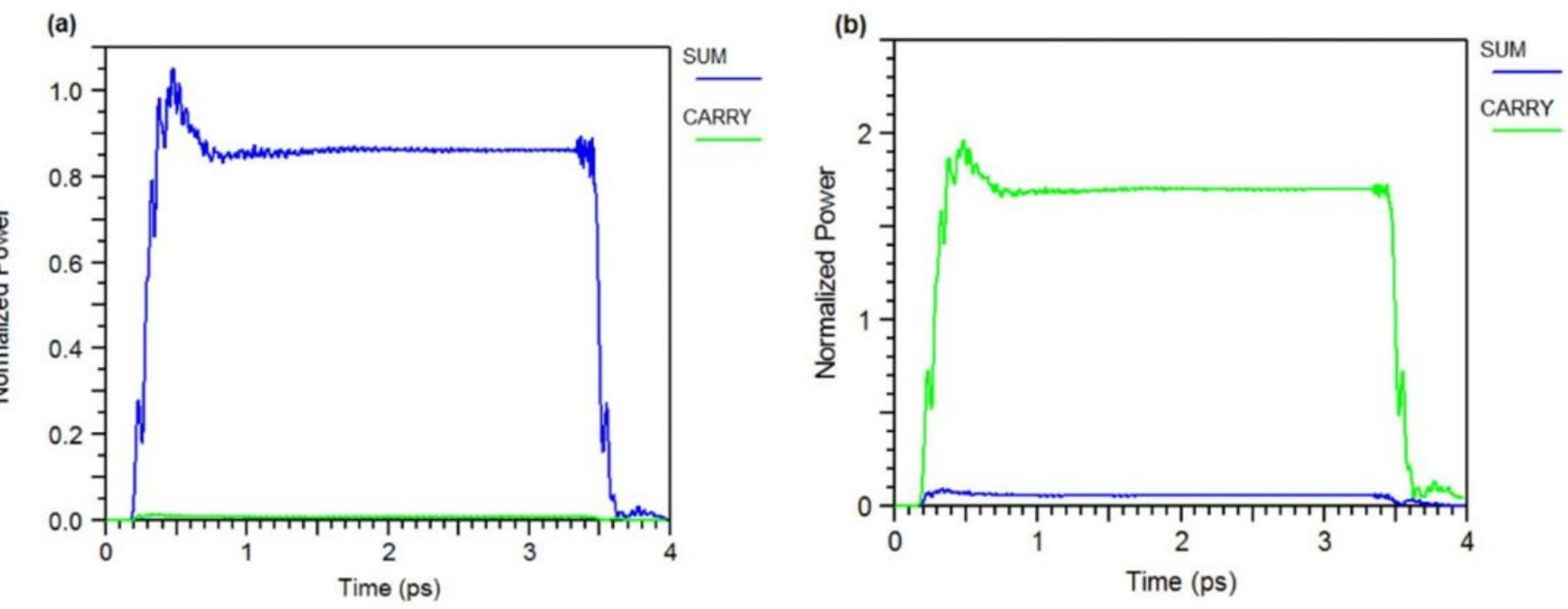

Figure 5

The proposed half subtractor's time response when (a) one and (b) two input ports are ON. 
(a)

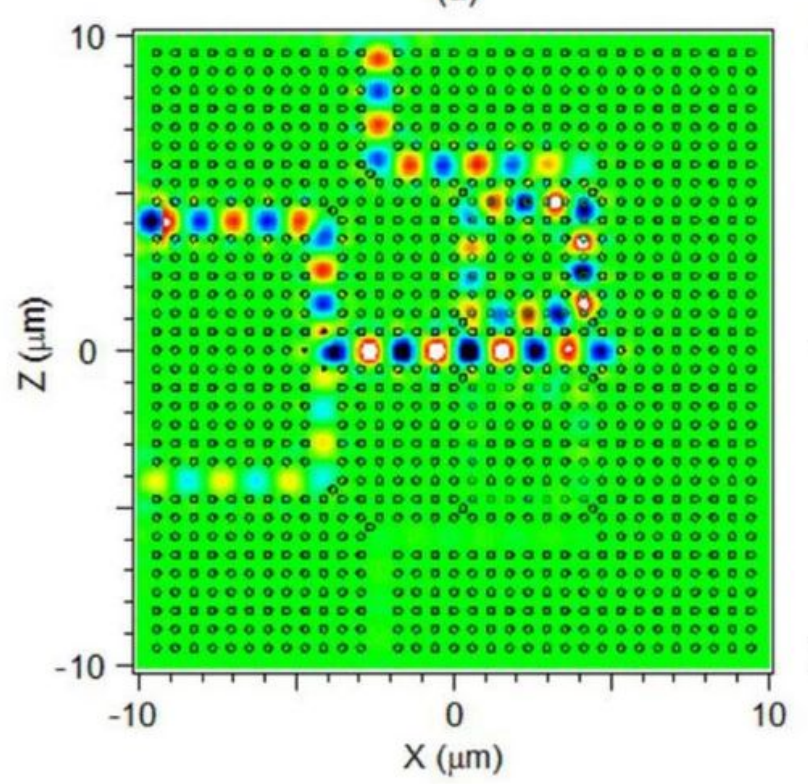

(b)

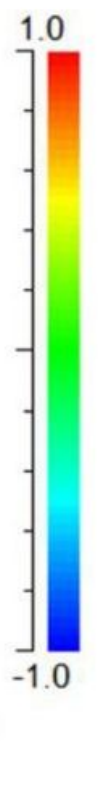

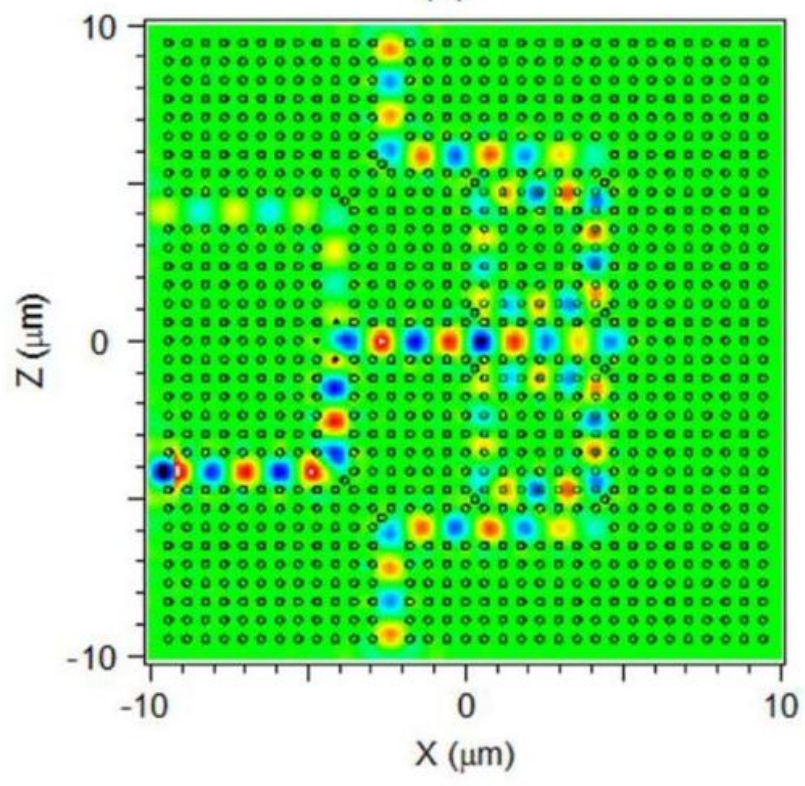

1.0

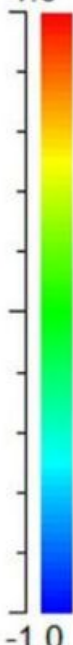

(c)

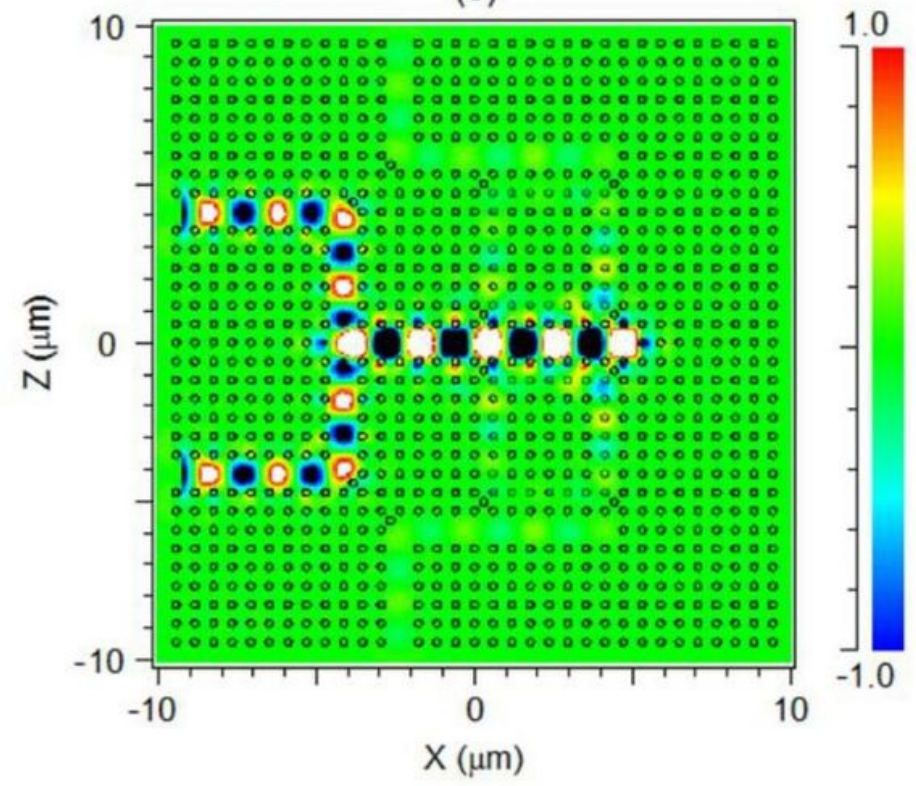

Figure 6

Light propagation inside half adder for (a) Case \#2 ( $X=1$ and $Y=0)$, (b) Case \#3 ( $X=0$ and $Y=1)$, and (c) Case \#4 ( $\mathrm{X}=1$ and $\mathrm{Y}=1)$. 

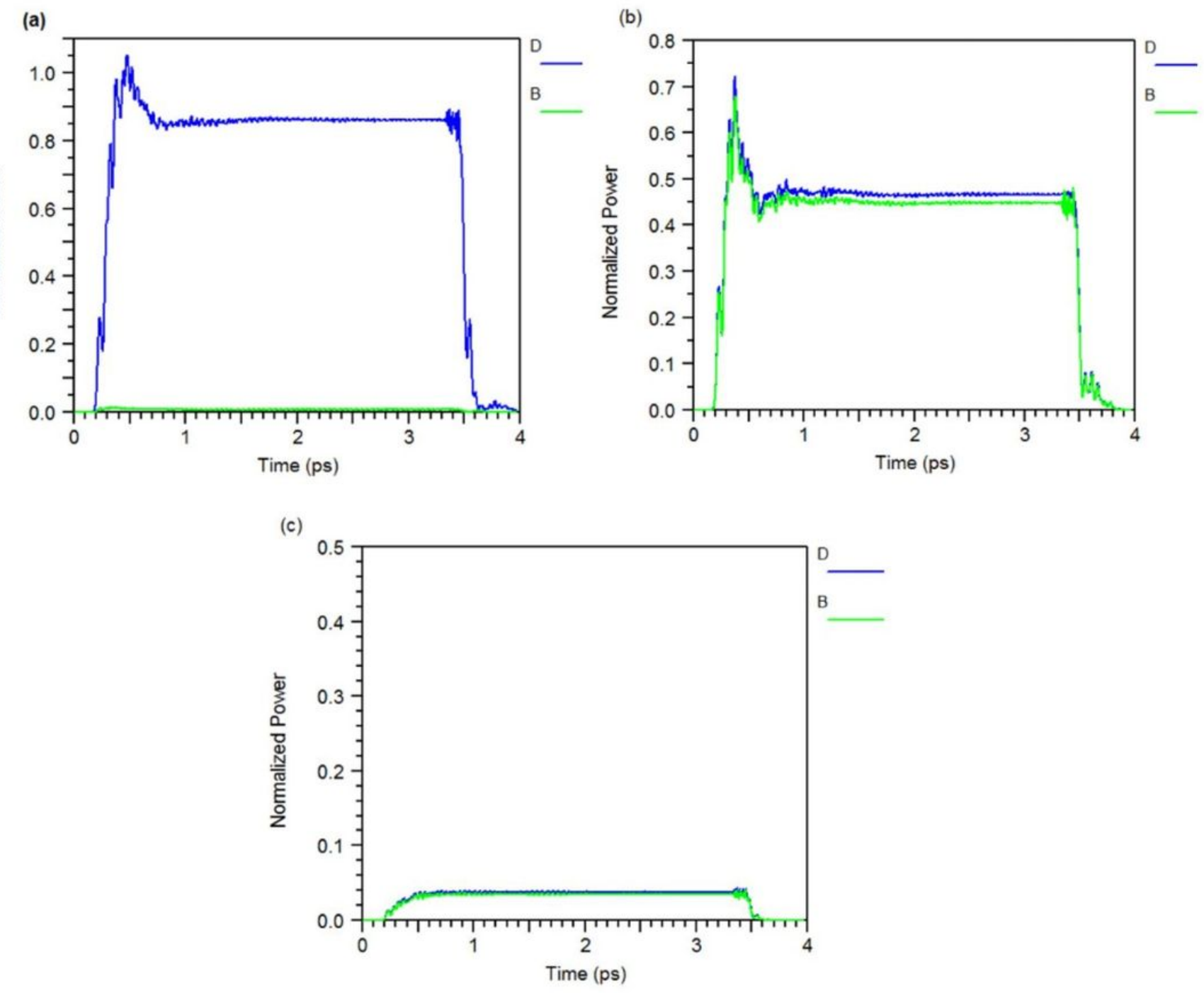

Figure 7

The time response of the proposed half adder for (a) Case \#2 ( $X=1$ and $Y=0)$, (b) Case \#3 ( $X=0$ and $Y=1)$, and (c) Case \#4 ( $\mathrm{X}=1$ and $\mathrm{Y}=1)$. 